\title{
Impact of Total Number of Lymph Node Retrieval on Patients with Esophageal Carcinoma Deserves Additional Investigation
}

\author{
Hanlu Zhang ${ }^{1} \cdot$ Yushang Yang ${ }^{1} \cdot$ Wenping Wang ${ }^{1} \cdot$ Yong Yuan $^{1} \cdot$ Yun Wang ${ }^{1} \cdot$ Long-Qi Chen $^{1}$
}

Received: 5 April 2019 / Accepted: 3 September 2019 / Published online: 12Septemb er2019

(C) 2019 The Society for Surgery of the Alimentary Tract

To the Editors:

We read with great interest the excellent article by Giugliano et al. in the Journal of Gastrointestinal Surgery. ${ }^{1}$ The authors found that there was no association between the total number of lymph node (TLN) retrieval and survival for patients with esophageal carcinoma after neoadjuvant chemoradiotherapy (nCRT) followed by surgery. Intriguingly, patients in the group of surgery alone showed the same result. We congratulate Giugliano et al. for this innovative and excellent study, but some points of the study warrant discussion.

The presence of lymph node metastasis is one of the most important predictors of survival in esophageal cancer patients with and without neoadjuvant chemoradiation. ${ }^{2}$ Resecting limited number of lymph nodes might cause stage migration, which incubated a doubt whether the study population was really "node-negative." Extensive lymphadenectomy not only contributes to accurately define $\mathrm{pN}+$ status but also improves survival outcomes. Both nCRT and an extensive lymphadenectomy could decrease potential micrometastatic nodes. Following neoadjuvant therapy, patients usually experience downstaging the primary tumor and decreasing micrometastases, so, for whom suboptimal lymphadenectomy might be enough to remove residual metastatic nodes. However, patients who are not responders to neoadjuvant therapy and those who are undergoing surgery alone are therefore more dependent upon an extensive lymphadenectomy to clear potential micrometastatic nodes. Therefore, the optimal number of lymph nodes (cutoff for comparison) obtained might be different for patients receiving upfront surgery and patients with neoadjuvant therapy. ${ }^{3}$

Though statistically nonsignificant $(p=0.07)$ in the nCRT group, the trend of survival benefit was observed in patients with

Long-Qi Chen

drchenlq@scu.edu.cn

1 Department of Thoracic Surgery, West China Hospital of Sichuan University, Chengdu 610041, China
TLN retrieval $\geq 15$ nodes than those with TLN retrieval of $<15$ (medial survival, 17.9 versus 35.1 months). Thus, we speculated more extensive lymphadenectomy was associated with improved survival for patients who received neoadjuvant chemoradiation followed by surgery. In our opinion, the cutoff of 15 lymph nodes by Giugliano et al. was arbitrary. A cut-point survival analysis could be used to determine the most significant statistical cutoff point for the number of resected lymph nodes. ${ }^{4}$

\section{Compliance with Ethical Standards}

Conflict of Interest The authors declare that they have no conflict of interest.

\section{References}

1. Giugliano DN, Berger AC, Pucci MJ, Rosato EL, Evans NR, Meidl H et al. Comparative Quantitative Lymph Node Assessment in Localized Esophageal Cancer Patients After R0 Resection With and Without Neoadjuvant Chemoradiation Therapy. Journal of gastrointestinal surgery : official journal of the Society for Surgery of the Alimentary Tract. 2017;21(9):1377-84. https://doi.org/10.1007/s11605-017-3478-y.

2. Mariette C, Piessen G, Briez N, Triboulet JP. The number of metastatic lymph nodes and the ratio between metastatic and examined lymph nodes are independent prognostic factors in esophageal cancer regardless of neoadjuvant chemoradiation or lymphadenectomy extent. Annals of surgery. 2008;247(2):365-71. https://doi.org/10. 1097/SLA.0b013e31815aaadf.

3. Samson P, Puri V, Broderick S, Patterson GA, Meyers B, Crabtree T. Extent of Lymphadenectomy Is Associated With Improved Overall Survival After Esophagectomy With or Without Induction Therapy. The Annals of thoracic surgery. 2017;103(2):406-15. https://doi.org/ 10.1016/j.athoracsur.2016.08.010.

4. Chen J, Chen $\mathrm{C}, \mathrm{He} \mathrm{Y}, \mathrm{Wu} \mathrm{K}, \mathrm{Wu} \mathrm{H}$, Cai S. A new pN staging system based on both the number and anatomic location of metastatic lymph nodes in gastric cancer. Journal of gastrointestinal surgery : official journal of the Society for Surgery of the Alimentary Tract. 2014;18(12):2080-8. https://doi.org/10.1007/s11605-014-2663-5.

Publisher's Note Springer Nature remains neutral with regard to jurisdictional claims in published maps and institutional affiliations. 